\title{
The Complementary Functions Method Solution to the Functionally Graded Polar Orthotropic Rotating Hyperbolic Disks with Both Radially and Circumferentially Aligned Fibers
}

\author{
Vebil Yıldırım \\ Department of Mechanical Engineering, Faculty of Engineering, University of Çukurova, Adana \\ E-mail address: vebil@cu.edu.tr \\ ORCID numbers of authors \\ 0000-0001-9955-8423
}

Received date: 11.11 .2018

Accepted date: 07.12.2018

\begin{abstract}
In the present study, efforts have been made to numerically evaluate elastic displacements and stresses in a convergent or divergent hyperbolic disk subjected to a centrifugal force of constant circular velocity. The disk material is assumed to be continuously radially functionally graded $(F G)$ with two orthotropic materials based on the simplest Voigt rule with a power of volume fraction of two constituents. The fibers are assumed to be aligned along either radial (RR) or circumferential (CR) directions. Having been a second order differential equation with variable coefficients, the governing equation so-called Navier equation is first derived and then put in the form of two differential equations of first order. These two ordinary differential equation set is originally solved based on the initial value problem (IVP) by employing the Complementary Functions Method (CFM). The numerical results are verified with the corresponding benchmark results for uniform thickness $F G$ polar orthotropic disks. The radial variation of the elastic fields in a hyperbolic disk is investigated for several boundary conditions, disk profile parameters, and the gradient parameter for both the radially and circumferentially aligned fibers. Some numerical results are also presented. Under the case that is considered in this study, it is revealed that the CR disk offers much higher elastic fields than RR disk under all boundary conditions. For a composite rotating disk rotating at a constant speed, it will be better to align fibers along the radial directions, to use convergent disk profiles, and to locate the material having higher radial stiffness at the outer surface. It is also disclosed that the location of the maximum Von-Mises equivalent stress in fixed-free disks varies regarding the fiber orientation.
\end{abstract}

Keywords: Anisotropic, complementary functions method, composite rotating disk, elasticity solution, functionally graded, initial value problem, polar orthotropic, variable-thickness, Voigt rule.

\section{Introduction}

Rotating disks are essential elements of turbine rotors, compressors, flywheels, automobile disc brake systems, gears, and etc. Today's scientific works focus on the use of advanced materials so that discs can withstand much higher rotational speeds and resulting stresses.

Rotating disk is a common component in diverse engineering applications such as turbine rotors, compressors, flywheels, disk brakes in automobiles, gears, computer disk drives, and etc. 
Anisotropic materials whose mechanical properties change in certain directions allow engineers to design and manufacture rotating disks that can withstand much higher critical/burst speeds than those made from common isotropic materials. As a new kind of advanced structural composite materials, FGMs have made considerable headway since the 1990s by virtue of their impeccable heat-resistance features. Despite the open literature contains many studies having very high academic standard on anisotropic disks, on FG metal-ceramic disks or the disks which are functionally graded with isotropic materials, any of them is not included in this article due to the space limitation.

Using anisotropic/orthotropic materials to form FG new kind nonhomogeneous materials is also one of the new trends in engineering design. There are, therefore, a limited number of works on FG disks composed of anisotropic materials in the open literature [1-8]. From those, Durodola and Attia [1] considered FG orthotropic materials to study deformation and stresses in rotating hollow uniform disks. Chen et al. [2] offered a three-dimensional analytical solution for a uniform rotating disk made of exponentially functionally graded materials with transverse isotropy. Kansal and Parvez [3] dealt with stress analysis on orthotropic graded rotating annular disks subjected to parabolic temperature distributions. Lubarda [4] analytically and numerically studied the elastic response of a uniformly pressurized cylindrically anisotropic hollow uniform thin rotating disks by using both the finite difference method and a Fredholm integral equation. Fredholm integral equation was also employed by Peng and Li [5] to consider FG hollow polarorthotropic rotating disks under free-free and fixed-free boundary conditions. Kacar and Yildırım [6] offered analytical formulas for the displacement and stress determination in polar orthotropic functionally power-law graded polar orthotropic rotating uniform disks under three boundary conditions. Essa and Argeso [7] developed analytical solutions for the analysis of elastic polar orthotropic FG annular free-free and fixed-free disks rotating with constant angular velocity. The elasticity moduli and thickness were assumed to be varied radially by a nonlinear function controlled by three parameters, while the radial variation of density may be defined by any form of continuous function. Essa and Argeso [7] also validated their analytical solutions by the use of a computational model based on the nonlinear shooting method. Based on the finite difference method and Voigt grading rule, Zheng et al. [8] numerically studied displacements and stress fields in a functionally graded fiber reinforced non-uniform thickness disk mounted on a rotating shaft and subjected to angular deceleration. The disk profile in the form of $(\alpha / r+\beta)$ and circumferentially reinforced fibers were considered in this comprehensive study. Zheng et al. [8] concluded that the disk deceleration has no effect on the radial and hoop stresses except the shear stress.

As seen from the open literature that there are few studies considered the CR-disks (Fig. 1) [812]. As far as the author knows, moreover, there is scarcely any comparative study on the elastic behavior of functionally graded CR and RR orthotropic disks. This was also a motivation for the author. 


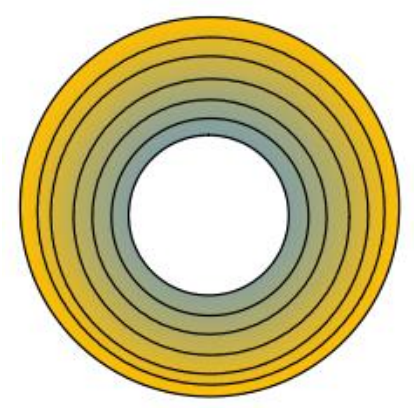

CR-disk

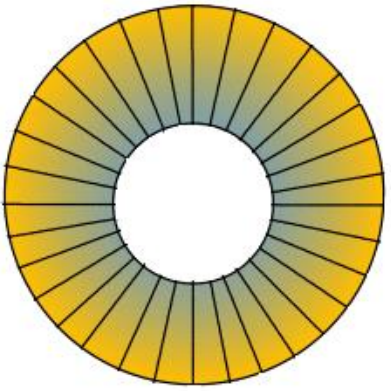

RR-disk

Fig. 1. Circumferentially and radially aligned disks

In this work, a comprehensive analysis that inspects the elastic characteristics of both RR and CR disks made of FG orthotropic materials and having either divergent or convergent hyperbolic disk profiles is presented (Figs. 1-2). A Voigt rule with a power of volume fraction of two orthotropic constituents is used to determine the radial variation of elastic constants. Poisson's ratios are taken to be constant. Within the frame of infinitesimal deformations and axisymmetric plane stress elasticity theory, Navier equation is derived and solved numerically based on the Complementary Functions Method (CFM) under three types of boundary conditions: free-free (traction-free inner and outer surfaces), fixed-free (rigidly circular shaftmounted inner surface and traction-free outer surface), and fixed-guided (rigidly circular shaftmounted inner surface and rigidly-cased outer surface). The present results are verified with the available literature [5].

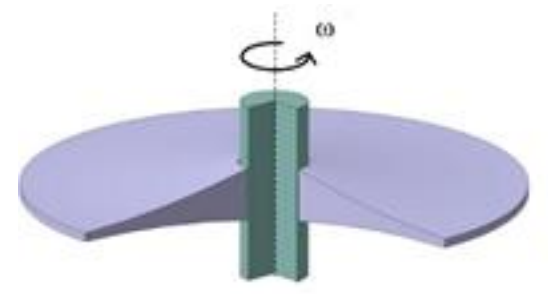

Convergent $(\mathrm{m}<0)$

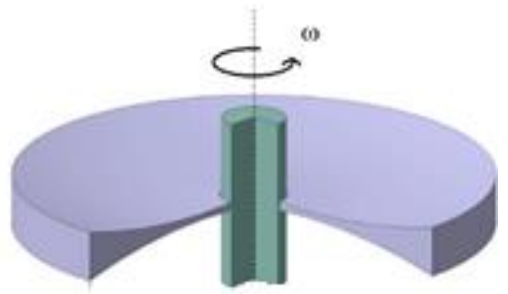

Divergent $(m>0)$

Fig. 2. Convergent and divergent hyperbolic disk profiles

\section{Derivation and Solution of Navier Equation}

Under small deformation assumptions, the strain-displacement relation is given by

$$
\varepsilon_{r}(r)=\frac{\mathrm{d} u_{r}(r)}{r}, \quad \varepsilon_{\theta}(r)=\frac{u_{r}(r)}{r}
$$

By presuming a state of axisymmetric plane stress (since the thickness/diameter ratio is far less than one) constitutive equation for RR-disk is to be (for CR disk, simply use $\left.E_{1}=E_{\theta}, E_{2}=E_{r}, v_{12}=v_{\theta r}\right)$ 


$$
\begin{gathered}
\begin{array}{c}
\sigma_{\mathrm{r}}(\mathrm{r})=-\frac{E_{\theta} v_{r \theta}}{v_{\theta r}\left(v_{r \theta} v_{\theta r}-1\right)} \varepsilon_{r}(r)-\frac{E_{\theta} v_{r \theta}}{\left(v_{r \theta} v_{\theta r}-1\right)} \varepsilon_{\theta}(\mathrm{r}) \\
=C_{11}(r) \varepsilon_{r}(r)+C_{12}(r) \varepsilon_{\theta}(r)
\end{array} \\
\begin{array}{c}
\sigma_{\theta}(r)=-\frac{E_{\theta} v_{r \theta}}{\left(v_{r \theta} v_{\theta r}-1\right)} \varepsilon_{r}(r)-\frac{E_{\theta}}{\left(v_{r \theta} v_{\theta r}-1\right)} \varepsilon_{\theta}(r)=C_{12}(r) \varepsilon_{r}(r)+C_{22}(r) \varepsilon_{\theta}(r)
\end{array}
\end{gathered}
$$

The equilibrium equation under the centrifugal forces is

$$
\left(h(r) r \sigma_{r}(r)\right)^{\prime}-h(r) \sigma_{\theta}(r)+\rho(r) h(r) \omega^{2} r^{2}=0
$$

Poisson's ratio are related by Maxwell's theorem as follows

$$
\frac{v_{\theta r}}{E_{\theta}(r)}=\frac{v_{r \theta}}{E_{r}(r)}
$$

Navier equation is derived from the field equations given above as follows

$$
\begin{aligned}
\frac{d^{2} u_{r}(r)}{d r} & +\left(\frac{1}{r}+\frac{\frac{d C_{11}(r)}{d r}}{C_{11}(r)}+\frac{\frac{d h(r)}{d r}}{h(r)}\right) \frac{d u_{r}(r)}{d r} \\
& +\left(-\frac{C_{22}(r)}{r^{2} C_{11}(r)}+\frac{C_{12}(r)}{r C_{11}(r)}\left(\frac{\frac{d C_{11}(r)}{d r}}{C_{11}(r)}+\frac{\frac{d h(r)}{d r}}{h(r)}\right)\right) u_{r}(\mathrm{r})=-\frac{\rho(r) \omega^{2} r}{C_{11}(r)}
\end{aligned}
$$

This equation is a second order differential equation with variable coefficients for boundary value problems (BVP). IVP form of the above Navier equation may be derived as follows

$$
\begin{gathered}
\frac{d u_{r}(r)}{d r}=-\frac{E_{\theta}(r) v_{r \theta}}{r E_{r}(r)} u_{r}(r)-\frac{\left(v_{r \theta} v_{\theta r}-1\right)}{E_{r}(r)} \sigma_{r}(r) \\
\frac{d \sigma_{r}(r)}{d r}=-\frac{E_{\theta}(r)\left(E_{r}(r)-E_{\theta}(r) v_{r \theta}^{2}\right)}{r^{2} E_{r}(r)\left(v_{r \theta} v_{\theta r}-1\right)} u_{r}(r) \\
+\left(\frac{E_{\theta}(r) v_{r \theta}}{r E_{r}(r)}-\frac{1}{r}-\frac{\frac{d h(r)}{d r}}{h(r)}\right) \sigma_{r}(r)-\rho(r) \omega^{2} r
\end{gathered}
$$

Equation (6) is written in a compact form of

$$
\frac{d \boldsymbol{Z}(r)}{d r}=\boldsymbol{D}(r) \boldsymbol{Z}(r)+\boldsymbol{f}(r)
$$

where 


$$
\begin{gathered}
\boldsymbol{Z}(r)=\left\{\begin{array}{l}
u_{r}(r) \\
\sigma_{r}(r)
\end{array}\right\}=\left\{\begin{array}{l}
z_{1}(r) \\
z_{2}(r)
\end{array}\right\} \\
\boldsymbol{f}(r)=\left\{\begin{array}{c}
0 \\
-\rho(r) \omega^{2} r
\end{array}\right\} \\
D_{11}=-\frac{E_{\theta}(r) v_{r \theta}}{r E_{r}(r)} \\
D_{12}=-\frac{\left(v_{r \theta} v_{\theta r}-1\right)}{E_{r}(r)} \\
D_{21}=-\frac{E_{\theta}(r)\left(E_{r}(r)-E_{\theta}(r) v_{r \theta}^{2}\right)}{r^{2} E_{r}(r)\left(v_{r \theta} v_{\theta r}-1\right)} \\
D_{22}=\frac{E_{\theta}(r) v_{r \theta}}{r E_{r}(r)}-\frac{1}{r}-\frac{d h(r)}{d r}
\end{gathered}
$$

The general solution of Eq. (7) in CFM over the interval [a, b] is given by [13-17]

$$
\begin{aligned}
& z_{1}(r)=x_{0}(r)+b_{1} x_{1}(r)+b_{2} x_{2}(r) \\
& z_{2}(r)=y_{0}(r)+b_{1} y_{1}(r)+b_{2} y_{2}(r)
\end{aligned}
$$

where unknown functions $x_{0}, x_{1}, x_{2}$ and $y_{0}, y_{1}, y_{2}$ are calculated by using those prescribed boundary conditions given in Table 1 in the first three stages of the method. At the final stage, the physical boundary conditions given in Table 2 are imposed in Eq. (9) to determine the remaining unknowns, $b_{1}$ and $b_{2}$. The solution has then been completed.

Table 1. Procedure for the first three steps of CFM

\begin{tabular}{lccc}
\hline Let & Solve Eq.(7) & $\begin{array}{l}\text { with the following prescribed } \\
\text { initial conditions }\end{array}$ & Find \\
\hline$\left\{\begin{array}{l}z_{1}=x_{0} \\
z_{2}=y_{0}\end{array}\right\}$ & with $\mathrm{f}(\mathrm{r}) \neq 0$ & $\left\{\begin{array}{l}z_{1}(\mathrm{a})=0 \\
z_{2}(\mathrm{a})=0\end{array}\right\}$ & $\left\{\begin{array}{l}x_{0} \\
y_{0}\end{array}\right\}$ \\
$\left\{\begin{array}{l}z_{1}=x_{1} \\
z_{2}=y_{1}\end{array}\right\}$ & with $\mathrm{f}(\mathrm{r})=0$ & $\left\{\begin{array}{l}z_{1}(\mathrm{a})=1 \\
z_{2}(\mathrm{a})=0\end{array}\right\}$ & $\left\{\begin{array}{l}x_{1} \\
y_{1}\end{array}\right\}$ \\
$\left\{\begin{array}{l}z_{1}=x_{2} \\
z_{2}=y_{2}\end{array}\right\}$ & with $\mathrm{f}(\mathrm{r})=0$ & $\left\{\begin{array}{l}z_{1}(\mathrm{a})=0 \\
z_{2}(\mathrm{a})=1\end{array}\right\}$ & $\left\{\begin{array}{l}x_{2} \\
y_{2}\end{array}\right\}$ \\
\hline
\end{tabular}


Table 2. Boundary conditions taken into consideration

\begin{tabular}{lcc} 
& $r=\mathrm{a}$ & $r=b$ \\
\hline Free-Free & $\sigma_{r}(a)=0$ & $\sigma_{r}(b)=0$ \\
Fixed-Free & $u_{r}(\mathrm{a})=0$ & $\sigma_{\mathrm{r}}(\mathrm{b})=0$ \\
Fixed-Guided & $u_{r}(\mathrm{a})=0$ & $u_{r}(\mathrm{~b})=0$ \\
\hline
\end{tabular}

\section{Material and Geometry of the Disk}

A hyperbolic disk profile function is determined as follows

$$
h(r)=h_{b}\left(\frac{r}{b}\right)^{m}
$$

In Eq. (10), $\mathrm{m}=0$ represents the uniform disk. Positive profile parameters offer divergent hyperbolic disks while the negative ones render convergent disks (Fig. 2).

In the present study, Voigt rule is employed with a power of volume fraction of constituents as follows [5]

$$
V_{B}=\left(\frac{r^{n}-a^{n}}{b^{n}-a^{n}}\right), n>0
$$

In this function (Fig. 3), the outer surface is to be Material B-rich (woven Glass fiber/Epoxy prepreg) while the inner surface is Materal A-rich (An injection molded Nylon 6 composite containing $40 \mathrm{wt} \%$ short glass fiber) (Table 3). 


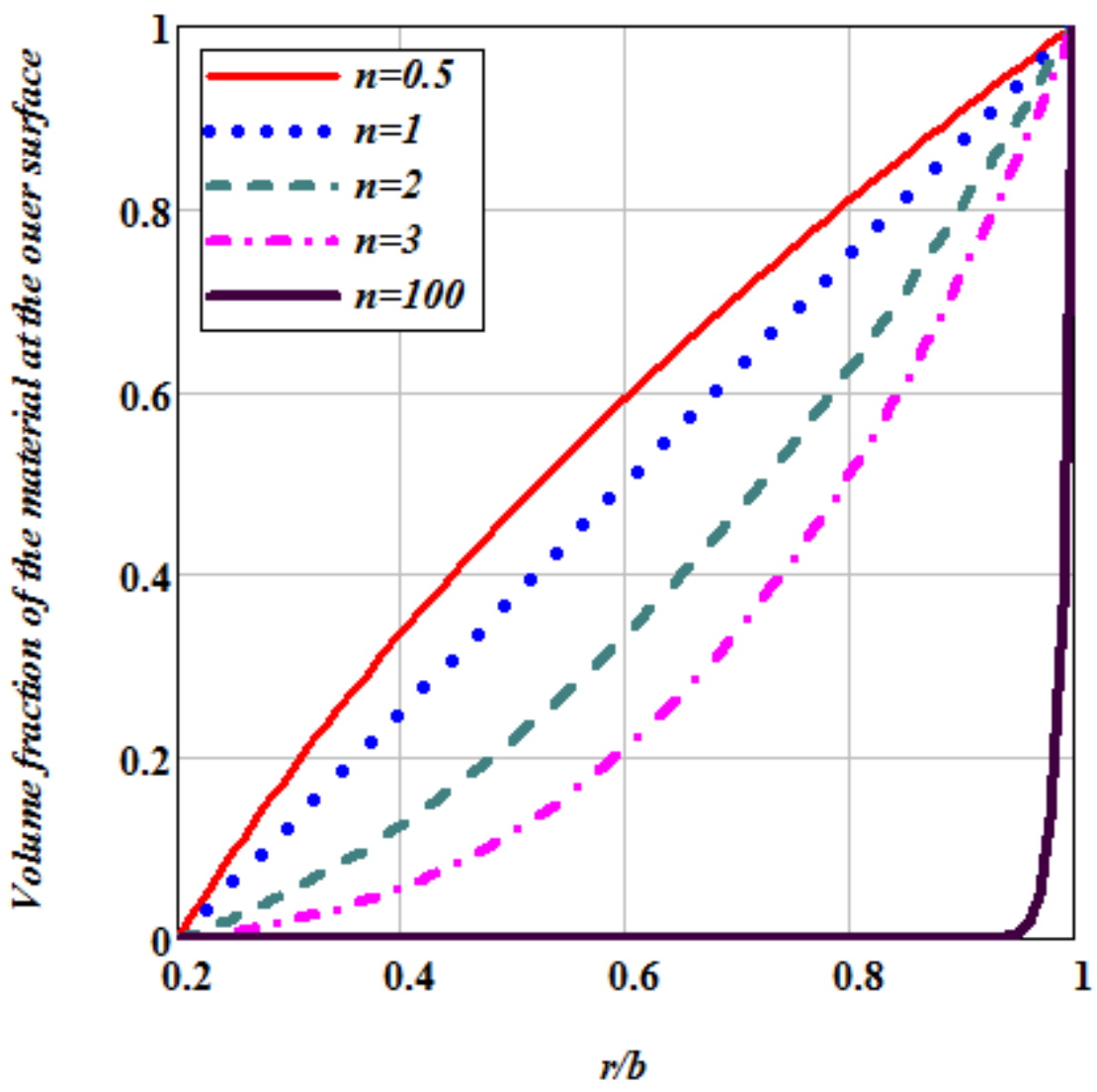

Fig. 3. Variation of volume fraction of the outer surface material

Table 3. Anisotropic materials and their properties.

\begin{tabular}{lcccc} 
& $E_{r}(\mathrm{GPa})$ & $E_{\theta}(\mathrm{GPa})$ & $\rho\left(\mathrm{kg} / \mathrm{m}^{3}\right)$ & \multicolumn{1}{c}{$v_{r \theta}$} \\
\hline Material-A [18] & 12.0 & 20.0 & 1600 & 0.21 \\
& & & & \\
Material-B [9] & 21.8 & 26.95 & 2030 & 0.15 \\
\hline
\end{tabular}

Based on the Voigt mixture rule, the radial variation of the effective material properties such as $E_{r}(r), E_{\theta}(r)$, and $\rho(r)$ are defined by

$$
P(r)=P_{A} V_{A}+P_{B} V_{B}=P_{A}\left(1-V_{B}\right)+P_{B} V_{B}=\left(P_{B}-P_{A}\right) V_{B}+P_{A}
$$

It is worth noting that, in the present numerical analysis, the arithmetic mean of Poisson's ratios of two orthotropic materials is considered.

$$
v_{r \theta}=\frac{v_{r \theta}^{A}+v_{r \theta}^{B}}{2}=\text { constant }
$$




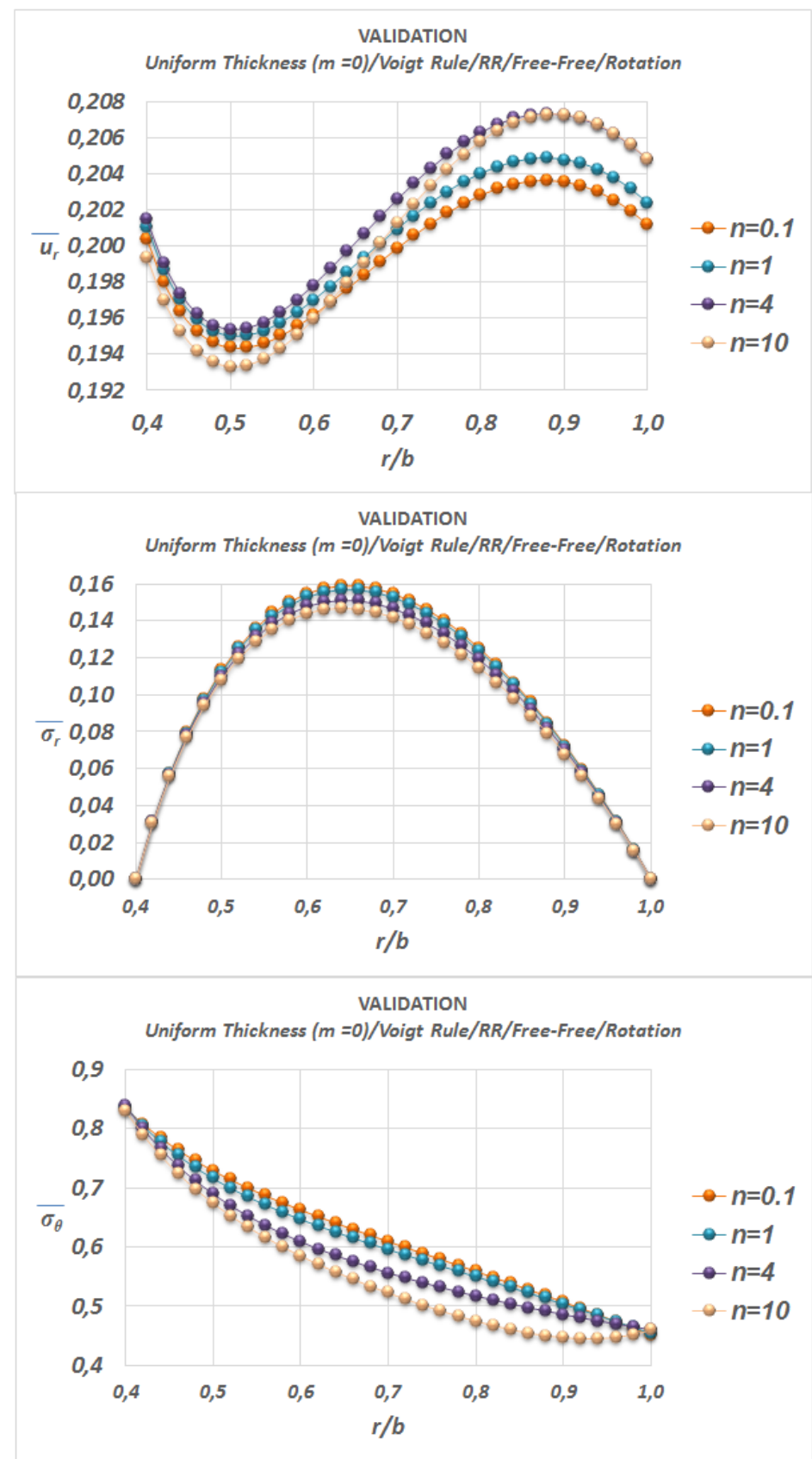

Fig. 4. Validation of the present results with Reference [5] $\left(\sigma_{o}=12 G P a . \rho_{o}=1600 \mathrm{~kg} / \mathrm{m}^{3}\right)$

\section{Numerical Study}

The dimensionless elastic fields are defined as

$$
\bar{u}_{r}=\frac{\mathrm{E}_{\mathrm{o}}}{\rho_{o} \omega^{2} b^{3}} u_{r}, \bar{\sigma}_{r}=\frac{\sigma_{\mathrm{r}}}{\rho_{o} \omega^{2} b^{2}}, \bar{\sigma}_{\theta}=\frac{\sigma_{\theta}}{\rho_{o} \omega^{2} b^{2}}
$$

To verify the present numerical results with material properties given in Table 3 , the example in Reference [5] is re-considered. Results are illustrated in Fig. 4. 
Comparison of the graphs in Fig. 4 and Reference [5] shows a good harmony although very minor differences are observed in the variation of the radial displacement. The reason of this that Peng and $\mathrm{Li}$ [5] used $v_{r \theta}=0.35=$ constant along the radial coordinate. As stated before, the arithmetic mean of Poisson's ratios is employed in the present study as in Eq. (13).
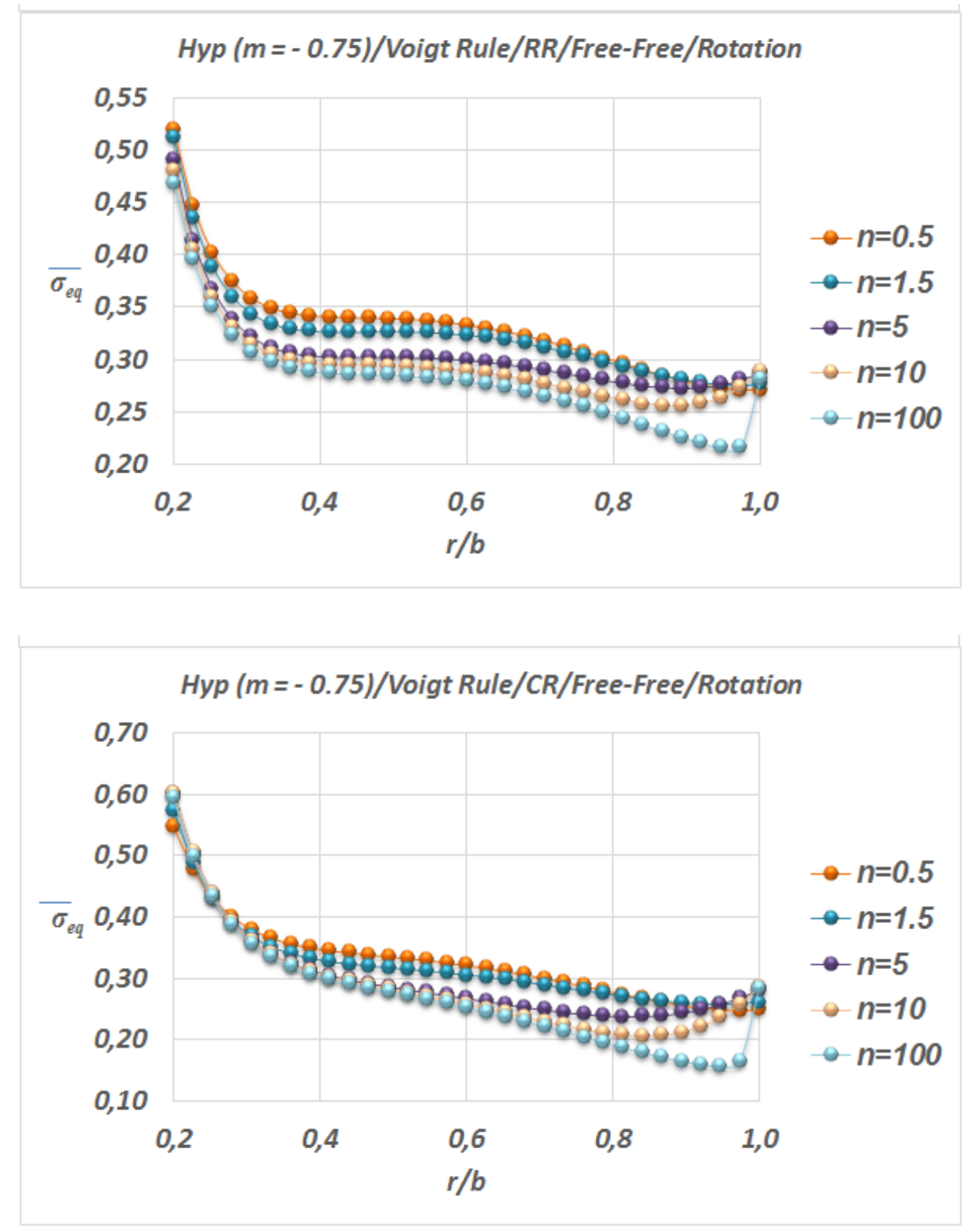

Fig. 5. Equivalent stress variation in a convergent free-free disk

Unless otherwise stated, $\sigma_{o}=20 G P a, \rho_{o}=1600 \mathrm{~kg} / \mathrm{m}^{3}$, a $/ \mathrm{b}=0.2$ and material properties in Table 3 are used in the other examples in this section. The following is also to be used for calculation of the equivalent von-Mises stresses.

$$
\sigma_{e q}=\sqrt{\sigma_{r}^{2}-\sigma_{r} \sigma_{\theta}+\sigma_{\theta}^{2}}
$$


Table 3. Elastic fields in a disk mounted a rigid shaft at its center for both RR-aligned and CR-aligned fibers (Fixed-Free $/ \mathrm{n}=1.5 / \sigma_{o}=20 \mathrm{GPa}, \rho_{o}=1600 \mathrm{~kg} / \mathrm{m}^{3}$ )

\begin{tabular}{|c|c|c|c|c|}
\hline $\mathrm{r} / \mathrm{b}$ & $\bar{u}_{r}$ & $\bar{\sigma}_{r}$ & $\sigma_{\theta}$ & $\bar{\sigma}_{e q}$ \\
\hline & \multicolumn{4}{|c|}{$m=-0.75$ (Convergent) - RR } \\
\hline 0.20 & 0.000000 & 0.254942 & 0.070393 & 200 \\
\hline 0.36 & 0.058276 & 0.267 & 0.239232 & 547 \\
\hline 0.52 & 0.109178 & 0.267832 & 0.298127 & 0.284193 \\
\hline 0.68 & 0.149197 & 0.226 & 0.310756 & 0.278384 \\
\hline 0.84 & 0.173387 & 0.138 & 0.289906 & 0.251152 \\
\hline \multirow[t]{2}{*}{1.00} & 0.178150 & 0.000000 & 0.240057 & 057 \\
\hline & \multicolumn{4}{|c|}{$m=-0.75$ (Convergent) $-\mathrm{CR}$} \\
\hline 0.20 & 0.000000 & 0.397176 & 0.069018 & 0.367560 \\
\hline 0.36 & 761 & & 0.16 & 570 \\
\hline 0.52 & 0.097730 & 0.32 & 0.206547 & 0.288594 \\
\hline 0.68 & 0.130795 & 0.260 & 0.218750 & 262 \\
\hline 0.84 & 0.1 & & 0.20 & 539 \\
\hline \multirow[t]{2}{*}{1.00} & 0.153441 & 0.0 & 0.167 & 250 \\
\hline & \multicolumn{4}{|c|}{$m=0$ (Uniform) $-\mathrm{RR}$} \\
\hline 0.20 & 0.000000 & 0.498896 & 52 & 61 \\
\hline 0.36 & 0.0 & & & 631 \\
\hline 0.52 & 0.1 & 0.31 & 678 & 0.36 \\
\hline 0.68 & 0.1 & 0.2 & 88 & 737 \\
\hline 0.84 & 0.2 & & 63 & 549 \\
\hline \multirow[t]{2}{*}{1.00} & 0.223040 & 0.000000 & 0.30 & 546 \\
\hline & \multicolumn{4}{|c|}{$m=0$ (Uniform) $-\mathrm{CR}$} \\
\hline 0.20 & 0.000000 & 0.857362 & 86 & 430 \\
\hline 0.36 & 0.0 & & 15 & \\
\hline 0.52 & 0.151229 & 0.400530 & 0.298017 & 0.360380 \\
\hline 0.68 & 0.1 & 0.2 & 0.2 & 745 \\
\hline 0.84 & 0.204963 & 405 & 0.26 & 0.234145 \\
\hline \multirow[t]{2}{*}{1.00} & 0.205853 & 0.000000 & 0.224380 & 0.224380 \\
\hline & \multicolumn{4}{|c|}{$m=0.75$ (Divergent) - RR } \\
\hline 0.20 & 0.000000 & 0.9 & 0.262785 & 0.851320 \\
\hline 0.36 & 0.145635 & 0.483175 & 0.549678 & 0.519628 \\
\hline 0.52 & 0.217426 & 0.347049 & 0.548290 & 397 \\
\hline 0.68 & 0.258985 & 0.24 & 0.504968 & 0.437422 \\
\hline 0.84 & 0.278433 & 0.131066 & 0.445852 & 0.396896 \\
\hline \multirow[t]{2}{*}{1.00} & 0.278505 & 0.000000 & 0.375286 & 0.375286 \\
\hline & \multicolumn{4}{|c|}{$m=0.75$ (Divergent) $-\mathrm{CR}$} \\
\hline 0.20 & 0.000000 & 1.809360 & 0.314418 & 1.674440 \\
\hline 0.36 & 0.162007 & 0.767196 & 0.442271 & 0.666997 \\
\hline 0.52 & 0.231863 & 0.464214 & 0.427418 & 0.446954 \\
\hline 0.68 & 0.267464 & 0.291406 & 0.396815 & 0.356013 \\
\hline 0.8 & 0.281906 & 0.147241 & 0.356954 & 0.310705 \\
\hline 1.00 & 0.279353 & 0.000000 & 0.304494 & 0.304494 \\
\hline
\end{tabular}



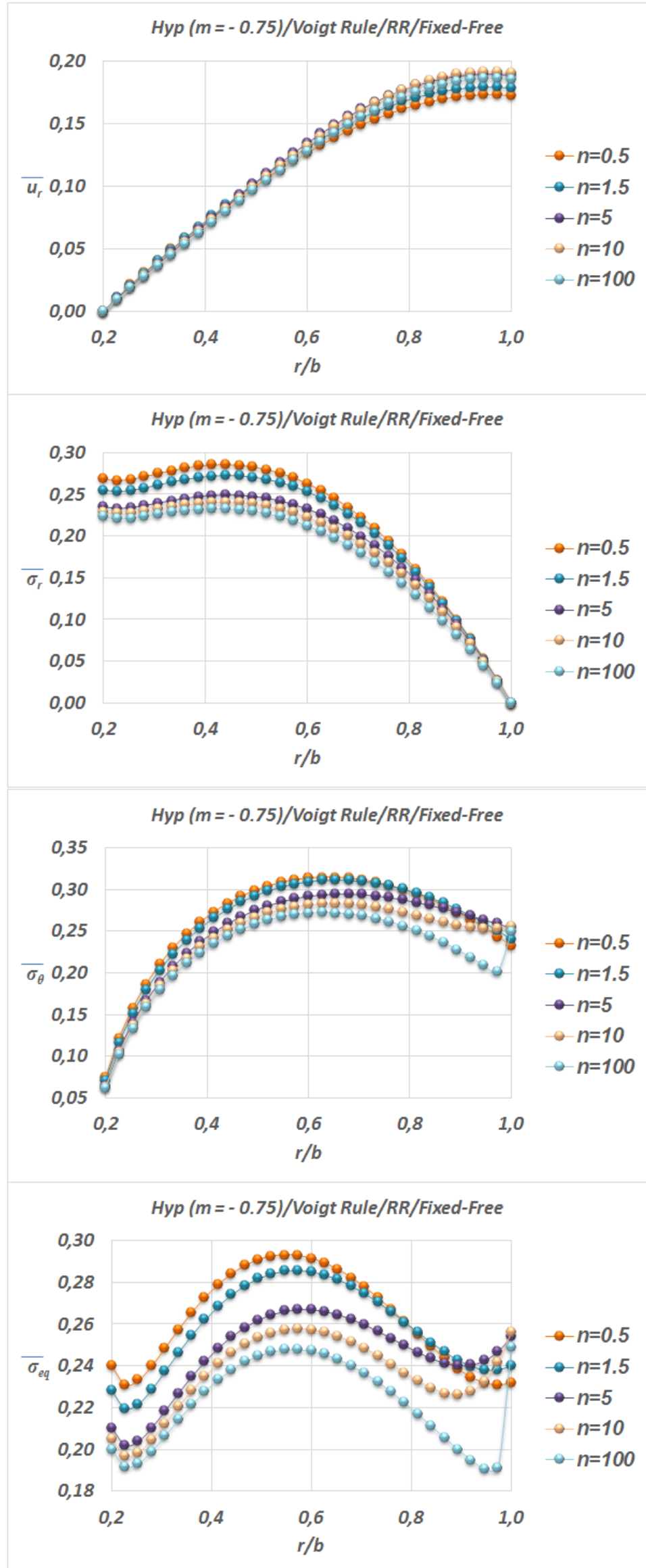

Fig. 6. Elastic fields in a convergent fixed-free RR-disk 
Results are presented in Table 3 and Figs. 5-9 for various cases. As explained before, Young's modulus in the radial direction of the outer surface material (Material-B) is assumed to be higher than the other. Table 3 reveals that the convergent hyperbolic profile and RR orientation are better than uniform and divergent ones since they offer smaller equivalent stresses under rotation and fixed-free boundary conditions. Figs. 5-9 suggest that RR disks have smaller equivalent stresses than CR ones under all boundary conditions. Fig. 6 and 8 disclosed that the location of the maximum equivalent stress in fixed-free disk depends on the fiber orientation.

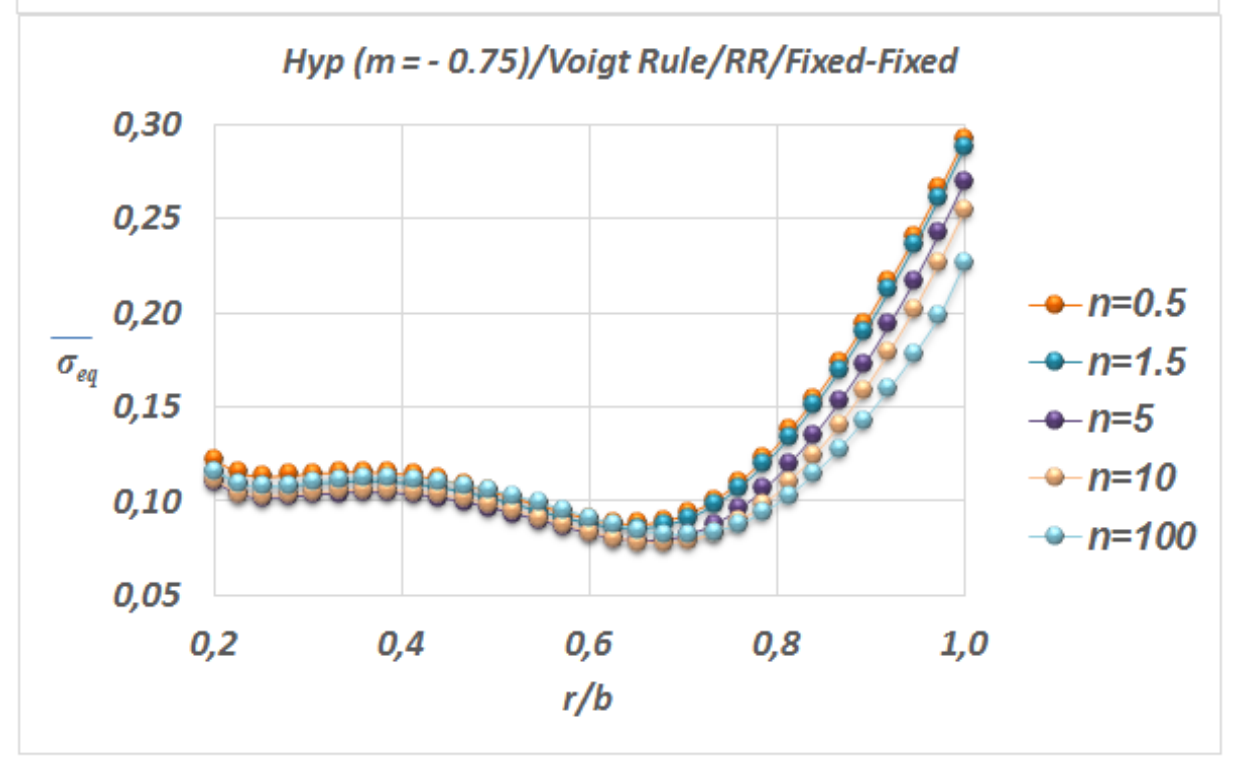

Fig. 7. Equivalent stress variation in a convergent fixed-fixed RR-disk

\section{Conclusions}

From the present study conducted with CFM the following results are achieved: i) CR-disks have higher equivalent stresses than RR-disks, ii) the location of the maximum Von-Mises equivalent stress in fixed-free disks depends on the fiber orientation, iii) if the outer surface material has higher radial stiffness than the inner surface material, a RR-disk having convergent profile has the smallest equivalent stresses than uniform and divergent ones under all boundary conditions.

\section{Nomenclature}

$a, b \quad:$ Inner and outer radii of the disk

$C_{i j} \quad$ : Stiffness components

$E(r) \quad$ : Effective Young's modulus

$E_{r}, E_{\theta}$ : Young's moduli along radial and tangential directions

$h(r) \quad$ : Disk profile function

$m \quad$ : Hyperbolic disk profile parameter

$n \quad:$ Gradation parameter

$r, \theta \quad:$ Radial and tangential coordinates

$u_{r} \quad$ : Radial displacement

$V \quad$ : Volume fraction 

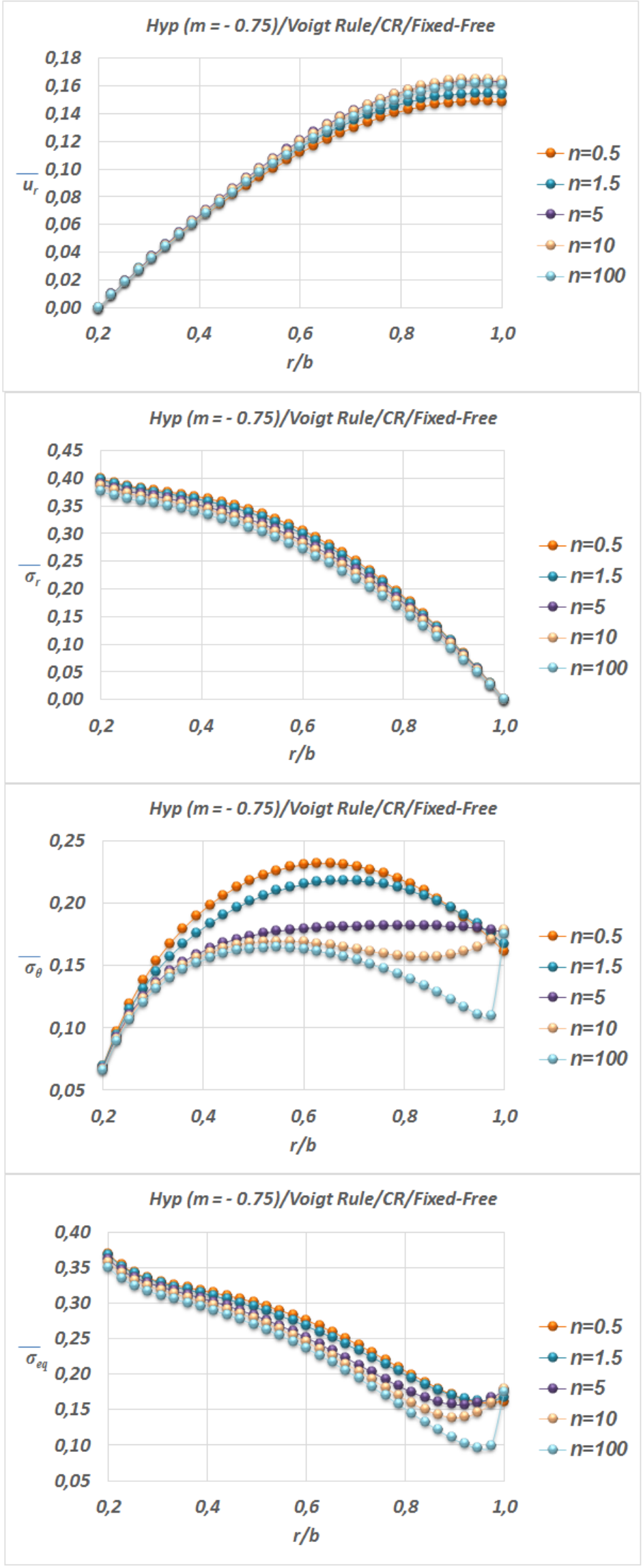

Fig. 8. Elastic fields in a convergent fixed-free CR-disk 


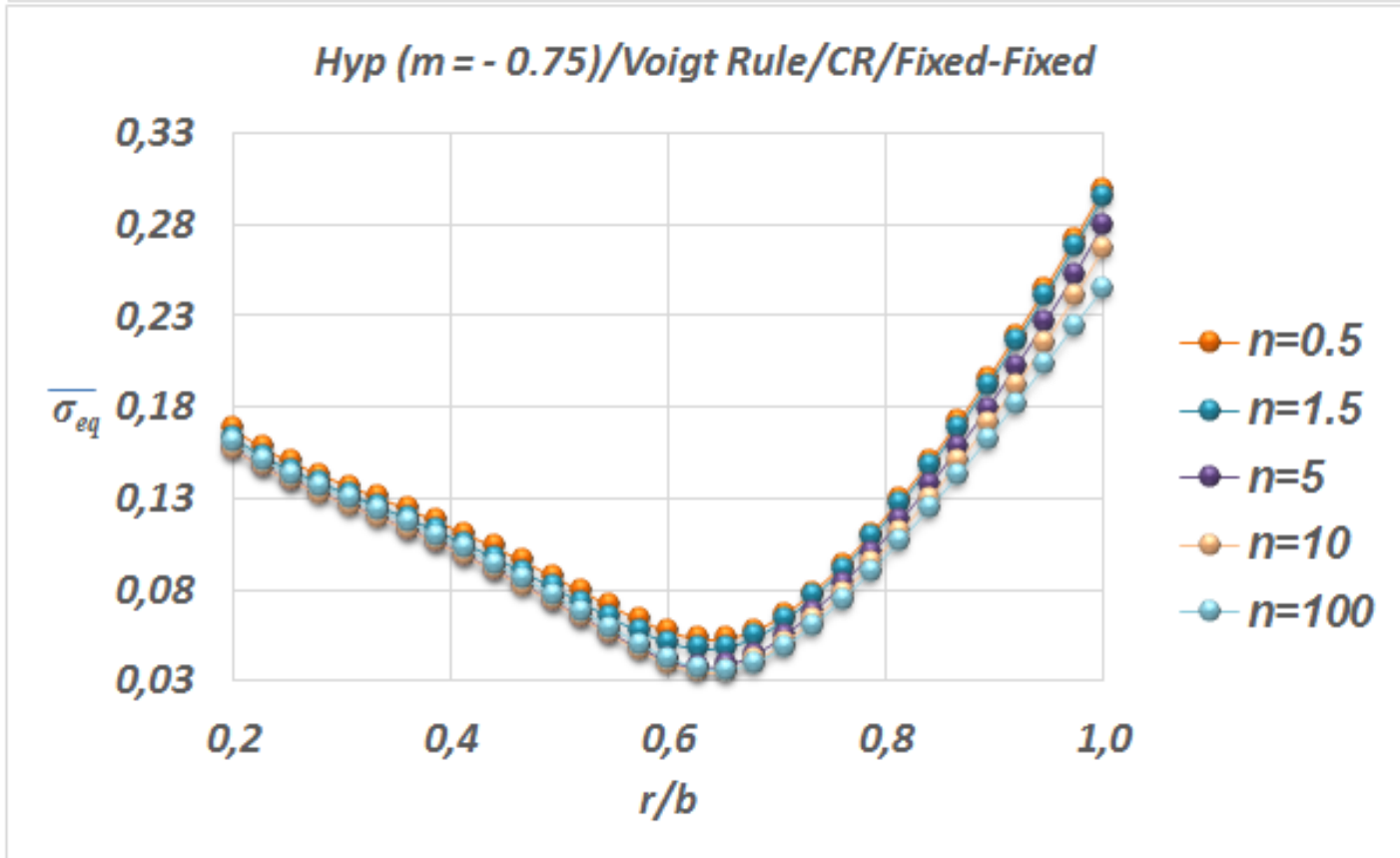

Fig. 9. Equivalent stress variation in a convergent fixed-fixed CR-disk

$\varepsilon_{r}, \varepsilon_{\theta}:$ Radial and tangential normal strain components

$\rho \quad:$ Material density

$\sigma_{e q} \quad$ : Equivalent Von-Mises stress

$\sigma_{r}, \sigma_{\theta} \quad$ : Normal radial and hoop stresses

$\omega \quad:$ Circular frequency

$v_{r \theta}, v_{\theta r}:$ Anisotropic Poisson's ratios

\section{Subcripts}

$a, A \quad$ : At the inner surface

$b, B \quad$ : At the outer surface

$o \quad:$ Reference value of the quantity

Overscripts

- $\quad$ : Dimensionless quantity

\section{References}

[1] Durodola, J., Attia, O., Deformation and stresses in functionally graded rotating discs", Compos Sci Technol, 60, 987-995, 2000.

[2] Chen, J., Ding, H., Chen, W., Three-dimensional analytical solution for a rotating disc of functionally graded materials with transverse isotropy, Archive of Applied Mechanics, 77, 241251, 2007.

[3] Kansal, G., Parvez, M., Thermal stress analysis of orthotropic graded rotating discs, International Journal of Modern Engineering Research (IJMER), 2(5), 3881-3885, 2012.

[4] Lubarda, V. A., On pressurized curvilinearly orthotropic circular disc, cylinder and sphere made of radially nonuniform material, Journal of Elasticity, 109, 103-133, 2012.

[5] Peng, X. L., Li, X. F., Elastic analysis of rotating functionally graded polar orthotropic discs, Int J Mech Sci, 60, 84-91, 2012. 
[6] Kacar, I., Y1ldırım, V., Effect of the anisotropy ratios on the exact elastic behavior of functionally power-graded polar orthotropic rotating uniform discs under various boundary conditions, Digital Proceeding of ICOCEE - Cappadocia 2017, Nevsehir, Turkey, 1743-1752, 2017.

[7] Essa, S., Argeso, H., Elastic analysis of variable profile and polar orthotropic FGM rotating disks for a variation function with three parameters, Acta Mech, 228, 3877-3899, 2017.

[8] Zheng, Y., Bahaloo, H., Mousanezhad, D., Vaziri, A., Nayeb-Hashemi, H., Displacement and stress fields in a functionally graded fiber-reinforced rotating disk with nonuniform thickness and variable angular velocity, J Eng Mater Technol, ASME, 39, 031010-1-9, 2017.

[9] Callioglu, H., Thermal stress analysis of curvilinearly orthotropic rotating discs, J Thermoplast Compos Mater, 20, 357-369, 2007.

[10] Callioglu, H., Topcu, M., Altan, G., Stress analysis of curvilinearly orthotropic rotating discs under mechanical and thermal loading, J Reinf Plast Compos, 24, 831-838, 2005.

[11] Tahani, M., Nosier, A., Zebarjad, S. M., Deformation and stress analysis of circumferentially fiber-reinforced composite disks, Int. J. Solids Struct, 42(9-10), 2741-2754, 2005.

[12] Zenkour A.M.,Allam N.M.N., On the rotating fiber-reinforced viscoelastic composite solid and annular disks of variable thickness, Int. J. Comput. Methods Eng. Sci Mech., 7, 21-31, 2006.

[13] Aktas, Z., Numerical Solutions of Two-Point Boundary Value Problems. Ankara, Turkey, METU, 1972.

[14] Roberts, S., Shipman, J., Fundamental matrix and two-point boundary-value problems, Journal of Optimization Theory and Applications, 28(1), 77-88, 1979.

[15] Haktanır, V., The complementary functions method for the element stiffness matrix of arbitrary spatial bars of helicoidal axes, Int J Numer Meth Eng, 38(6), 1031-1056, 1995.

[16] Yildırım, V., Free vibration analysis of non-cylindrical coil springs by combined use of the transfer matrix and the complementary functions methods, Comm Numer Meth Eng, 13(6), 48794, 1997.

[17] Yildırım, V., The complementary functions method (CFM) solution to the elastic analysis of polar orthotropic rotating discs, J. Appl. Comput. Mech, 4(3), 216-230, 2018. DOI: 10.22055/JACM.2017.23188.1150

[18] Callioglu, H., Stress analysis of an orthotropic rotating disc under thermal loading, J Reinf Plast Compos, 23(17), 1857-1869, 2004. 